\title{
Effects of individualized high positive end-expiratory pressure and crystalloid administration on postoperative pulmonary function in patients undergoing robotic-assisted radical prostatectomy: a prospective randomized single-blinded pilot study
}

Sebastian Blecha ( $\sim$ Sebastian.Blecha@ukr.de)

University Medical Centre Regensburg

Anna Hager

University Medical Centre Regensburg

Verena Gross

University Medical Centre Regensburg

Timo Seyfried

University Medical Centre Regensburg

Florian Zeman

University Medical Centre Regensburg

Matthias Lubnow

University Medical Centre Regensburg

Maximilian Burger

University Medical Centre Regensburg

Michael Pawlik

University Medical Centre Regensburg

\section{Research Article}

Keywords: fluid management, individual PEEP, pulmonary function, robotic-assisted laparoscopic prostatectomy, steep Trendelenburg position

Posted Date: July 29th, 2021

DOI: https://doi.org/10.21203/rs.3.rs-625423/v1

License: (c) (i) This work is licensed under a Creative Commons Attribution 4.0 International License.

Read Full License 


\section{Abstract}

Background Robotic-assisted laparoscopic prostatectomy (RALP) using a combination of capnoperitoneum and steep Trendelenburg positioning (STP) results in important pathophysiological pulmonary changes. The aim of the study was to evaluate if restrictive crystalloid administration and individual management of positive end-expiratory pressure (PEEP) improve peri- and postoperative pulmonary function in patients undergoing RALP in permanent 45 degree STP.

Methods 98 patients undergoing RALP under standardized anesthesia were either allocated to a standard PEEP ( $5 \mathrm{mmHg}$ ) group or an individualized high PEEP group. Furthermore, each group was divided into a liberal vs restrictive crystalloid group ( $30 \mathrm{ml}$ vs $15 \mathrm{ml}$ per $\mathrm{kg}$ predicted body weight). Individualized PEEP levels were determined by means of preoperative PEEP titration in STP. In each of the four study groups following intraoperative parameters were analyzed: ventilation setting (PIP, driving pressure $\left[\mathrm{P}_{\text {driv }}\right]$, lung compliance [LC], mechanical power [MP]), and postoperative pulmonary function (bed-side spirometry). The following spirometric parameters were measured pre- and postoperatively: the Tiffeneau index $\left(\mathrm{FEV}_{1} / \mathrm{FVC}\right.$ ratio) and mean forced expiratory flow $\left(\mathrm{FEF}_{25}-75\right)$. Data are shown as mean \pm standard deviation (SD), and groups were compared with ANOVA. A P-value of $<0.05$ was considered significant.

Results The two individualized high PEEP groups (mean PEEP $15.5\left[ \pm 1.71 \mathrm{cmH}_{2} \mathrm{O}\right]$ ) showed significantly higher PIP and MP levels but significantly decreased $P_{\text {driv }}$ and increased LC. On the first and second postoperative day, patients with individualized higher PEEP levels had a significantly higher mean Tiffeneau index (day 1: $77.6 \%( \pm 6.6)$ vs $73.6 \%( \pm 8.8), P=0.014$; day $2: 76.5 \%( \pm 6.1)$ vs $72.7 \%( \pm 9.3), P=$ 0.021 ) and $\mathrm{FEF}_{25-75}$ (day 1: 2.41 liter/sec $( \pm 0.9)$ vs 1.95 liter/sec $( \pm 0.8), P=0.009 ;$ day $2: 2.45$ liter/sec $( \pm 0.9)$ vs 2.07 liter/sec $( \pm 0.8), P=0.033)$. Perioperative oxygenation and postoperative spirometric parameters were not influenced by restrictive or liberal crystalloid infusion in either of the two PEEP groups.

Conclusions Higher individualized PEEP levels during RALP improved blood oxygenation, lung-protective ventilation, and postoperative pulmonary function up to 48 hours after surgery. Restrictive crystalloid infusion during RALP seemed to have no effect on peri- and postoperative oxygenation and pulmonary function.

\section{Trial Registration}

German Clinical Trials Register (DRKS00016887, prospectively registered 07/03/2019)

URL: https://www.drks.de/drks_web/setLocale_EN.do

\section{Introduction}

Robotic-assisted laparoscopic prostatectomy (RALP) has been the fastest-developing surgical technique for treating prostate carcinoma in recent years. RALP has many advantages over open surgery, including 
minimal tissue trauma, lower blood loss and transfusion rates, fewer surgical and postoperative complications, earlier postoperative recovery, and improved functional results [1-3]. RALP requires capnoperitoneum and steep Trendelenburg positioning (STP) with a head-down tilt of at least 35-45 degrees. The necessity of having to maintain adequate ventilation and normal $\mathrm{CO}_{2}$ levels in patients undergoing RALP makes it difficult to avoid high minute ventilation with increased peak inspiratory pressure (PIP) [4, 5]. Ventilation decreases lung movement, resulting in the elevation of the diaphragm. Many anaesthesiologists increase the PEEP level to prevent the collapse of the airways. The aim of a 'best PEEP concept' is to detect the optimal individual PEEP to enable non-harming ventilation for lung compliance (LC) and to minimize the development of mechanical lesions due to ventilation [6].

In the last twenty years, much knowledge has been gained on the lung physiology of both healthy and critically ill patients. Limited high plateau pressure and tidal volume $\left(\mathrm{V}_{T}\right)$, appropriate positive endexpiratory pressure (PEEP), restriction of fluid, and adequate driving pressure $\left(P_{\text {driv }}\right)$ in the lung (i.e. plateau pressure - PEEP) have resulted in fewer days of ventilation and better survival rates in many countries, at least in critically ill patients with acute respiratory distress syndrome (ARDS) [7]. In 2015, Amato et al. described the association of $\mathrm{P}_{\text {driv }}$ with mortality in patients with ARDS, and this report has led to a different perspective on ventilation during anaesthesia, even in healthy patients[8]. Furthermore, protective ventilation with lower $\mathrm{V}_{\mathrm{T}}$ has been associated with better clinical outcome, even in patients without ARDS[9]. Moreover, stressed lungs are prone to collect fluid in the interstitial space, leading to a decline in oxygen saturation and postoperative lung functions tests[10]. In patients with an ARDS, a restrictive strategy of fluid management improved lung function and shortened the duration of mechanical ventilation and intensive care without increasing nonpulmonary-organ failures [11, 12]. Furthermore, in patients undergoing colorectal surgery, a restricted perioperative intravenous fluid regime reduces postoperative complications[13]. Otherwise, a current study showed that a restrictive fluid regimen was associated with a higher rate of acute kidney injury in patients with major abdominal surgery[14]. Currently, the best fluid regime for patients undergoing RALP is unknown.

In this study, patients with prostate cancer undergoing RALP were allocated either to a standard PEEP (5 $\mathrm{cmH}_{2} \mathrm{O}$ ) group or an optimal PEEP group. Additionally, we analysed the effects of both liberal and restrictive fluid management on pulmonary function up to 48 hours after RALP. The primary hypothesis was that the combination of individualized high PEEP values and restrictive administration of crystalloids increases postoperative pulmonary function and improves perioperative oxygenation and ventilation in patients undergoing RALP.

\section{Methods}

\section{Study design}

This prospective, randomized, single-centre, and single-blinded pilot-study was approved by the local institutional review board (Protocol no. 18-1224-101, approved on 12 December 2018) and registered with 
the German Clinical Trials Register (DRKS00016887, prospectively registered on 07/03/2019). Informed consent was obtained from 104 patients scheduled for elective prostatectomy at the Department of Urology at the Caritas St. Josef University Medical Centre Regensburg. All patients were recruited between March 2019 and October 2019. Main exclusion criteria were pre-existing pulmonary disease (bronchial asthma, chronic obstructive pulmonary disease [COPD]), age $>80$ years, BMl $>35 \mathrm{~kg} / \mathrm{m}^{2}$, American Society of Anaesthesia physical status $>$ III, known cardiac insufficiency, and pulmonary hypertension. Randomization was done by the Centre for Clinical Studies. A randomization list was generated using SAS 9.4 (SAS Institute Inc., Cary, NC, USA) and the procedure plan (block-wise randomization with a block length of 8). Sequentially numbered opaque randomization envelopes containing patient assignment to one of the study groups were generated by the Centre for Clinical Studies and stored at the University Department of Anaesthesiology. An envelope was opened by the principal investigator (MP) after the patient had signed the informed consent.

\section{Patient cohort}

Figure 1 depicts an overview of the sample size of the study. The study preliminarily included 104 patients with prostate cancer who had been treated at the Department of Urology between March 2019 and October 2019. The patients were preoperatively randomized to one of the four study groups (Figs. 1 and 2): standard or individualized PEEP with administration of restrictive or liberal crystalloids. Six patients were excluded from analysis because of missing data, study protocol violations (missing of ventilation and/or spirometry settings [ $n=5]$, or retroactive withdrawal of consent $[n=1]$ ).

\section{Anaesthesia protocol and surgical technique}

The anaesthesia protocol, standardized for drug administration during RALP, was exclusively conducted by three anaesthesiologists throughout the entire study. These three anaesthesiologists were independent and not included in the analysis and publication of this study. Drug dosing was based on the calculated predicted body weight (PBW formula: PBW [men] $=50+0.91$ * [cm of height -152.4$]$ in $\mathrm{kg}$ ) [15]. Anaesthesia was induced with sufentanil (initial $0.5 \mu \mathrm{g} / \mathrm{kg}$ bolus), propofol (2-3 mg/ $\mathrm{kg}$ ), and atracurium $(0.5 \mathrm{mg} / \mathrm{kg})$. After tracheal intubation with a 7.5 or $8.0 \mathrm{~mm}$ endotracheal tube, anaesthesia was maintained with sufentanil (repetition of $10 \mu \mathrm{g}$ every 30 to $45 \mathrm{~min}$ until $30 \mathrm{~min}$ before end of surgery) and propofol (4-6 mg/kg/h) as total intravenous anaesthesia (TIVA). TIVA was used as standard anaesthesia for RALP to reduce postoperative nausea and vomiting and to minimize the influence on pulmonary function by volatile anaesthetics. After endotracheal intubation, the individualized PEEP arm received a decremental PEEP titration in STP, during which PEEP was set to $22 \mathrm{cmH}_{2} \mathrm{O}$ and decreased stepwise by $2 \mathrm{cmH}_{2} \mathrm{O}$ every $3 \mathrm{~min}$. At each PEEP step, the best compliance value was observed. This individual PEEP was maintained throughout mechanical ventilation. No recruitment maneuvers were employed in either group. All patients received volume-controlled ventilation with PEEP based on the respective group, using an inspiration-to-expiration ratio of 1:1, a basic respiratory rate of 12 , and a constant $\mathrm{V}_{\mathrm{T}}$ of 8-9 $\mathrm{ml} / \mathrm{kg}$ PBW. Pneumoperitoneum was created by intraperitoneal insufflation of $\mathrm{CO}_{2}$ to a standard value of $15 \mathrm{mmHg}$ with the patient in supine position. Each patient was consequently placed 
in a 45-degree STP. Surgery was always exclusively conducted by three urologists. High individualized PEEP values were reduced to $8 \mathrm{cmH}_{2} \mathrm{O}$ at the end of surgery after positioning the patient in a supine position prior to extubation.

\section{Measurement of perioperative ventilation and blood oxygenation}

All patients were ventilated with the Perseus ${ }^{\circledR}$ Ventilator (Dräger Medical, Lübeck, Germany). Lung parameters and blood oxygenation were measured in each patient at predefined time points (Fig. 2). The following respiratory parameters were documented during mechanical ventilation: fraction of inspired oxygen $\left(\mathrm{F}_{\mathrm{i}} \mathrm{O}_{2}\right)$, minute volume $(\mathrm{MV})$, respiratory rate, peak inspiratory pressure $(\mathrm{PIP})$, plateau pressure, PEEP, dynamic LC, and end-tidal $\mathrm{CO}_{2}$. Blood gases were analysed with the radiometer $\mathrm{ABL} 800 \mathrm{Flex}$ (Radiometer Medical, Copenhagen, Denmark). The following parameters were investigated: $\mathrm{pH}$ value, partial pressure of arterial oxygen $\left(\mathrm{P}_{\mathrm{a}} \mathrm{O}_{2}\right)$, partial pressure of arterial carbon dioxide $\left(\mathrm{PaCO}_{2}\right)$, arterial oxygen $\left(\mathrm{SaO}_{2}\right)$, and base excess $(\mathrm{BE})$. The $\mathrm{P}_{\mathrm{a}} \mathrm{O}_{2} / \mathrm{F}_{\mathrm{i}} \mathrm{O}_{2}$ ratio (also referred to as $\mathrm{P} / \mathrm{F}$ ratio or Horovitz index) was calculated based on $\mathrm{PaO}_{2}$ and applied $\mathrm{FiO}_{2}$. The $\mathrm{P} / \mathrm{F}$ ratio, which is used for assessing pulmonary function in ventilated patients, is an indicator for hypoxemia. Additionally, we calculated driving pressure $\left(P_{\text {driv }}\right)$ as the difference between plateau pressure and PEEP and the mechanical power $(\mathrm{MP})$ with the following formula: MP (in $\mathrm{J} / \mathrm{min})=0.098 * \mathrm{~V}_{\mathrm{T}} * \mathrm{RR} *\left(\mathrm{PIP}-0,5 * \mathrm{P}_{\text {driv }}\right)[16,17]$.

\section{Measurement of postoperative pulmonary function, body weight, and brain natriuretic peptide}

Spirometry was carried out with the Vitalograph Micro® (Vitalograph GmbH, Hamburg, Germany) in sitting position at the following four time points: on the day before surgery, 60 minutes after extubation in the recovery room, and at the first and second postoperative day. Two physicians (VG, AH) observed each spirometry, received technical instruction by the manufacturer, and were briefed by a pulmonologist (ML) in using the spirometer. The physicians were blinded for the pulmonary function measurement, i.e. they did not know in which group the patients were randomized. The following parameters were measured with the spirometer: vital capacity (VC), forced vital capacity (FVC), forced expiratory volume in one second $\left(\mathrm{FEV}_{1}\right)$, the Tiffeneau index ( $F E V_{1} / F V C$ ratio), peak expiratory flow (PEF), forced expiratory flow after one quarter of FVC $\left(\mathrm{FEF}_{25}\right)$, forced expiratory flow after half of FVC $\left(\mathrm{FEF}_{50}\right)$, forced expiratory flow after three quarter of FVC $\left(\mathrm{FEF}_{75}\right)$, and mean forced expiratory flow $\left(\mathrm{FEF}_{25-75}\right)$. Additionally, body weight and brain natriuretic peptide (BNP) were established preoperatively and on the first and second postoperative day. A normal BNP level was defined as less than 35 pg/ml [18].

\section{Study aims}

The primary aim of the study was to investigate thy dynamic of different spirometric parameters (VC, $\mathrm{FVC}, \mathrm{FEV}_{1}, \mathrm{FEV}_{1} / \mathrm{FVC}$ ratio, $\mathrm{PEF}_{1} \mathrm{FEF}_{25}, \mathrm{FEF}_{50}, \mathrm{FEF}_{75}$, and $\mathrm{FEF}_{25-75}$ ) of the postoperative pulmonary function in the recovery room up to the second postoperative day differentiated between the four different 
study groups. Secondary aims included parameters regarding perioperative ventilation and blood oxygenation.

\section{Statistics}

Sample size considerations

This study was designed as a pilot study with several primary variables to get insights about postoperative pulmonary function dependent on standard or individualized PEEP and on administration of restrictive or liberal crystalloids. Thus, no a priori sample size calculation could be performed. Since we expected small to medium effect sizes, a sample size of $n=25$ per group $\left(n_{\text {total }}=100\right)$ was targeted to get rather robust effect estimates in all four groups for planning of further studies [19].

Statistical methods

Data are shown as mean \pm SD for continuous variables and as absolute and relative frequencies for categorical variables. All continuous variables were compared between two or more groups by using an Analysis of Variance (ANOVA). All reported P-values are two-sided, and a P-value of 0.05 was considered the threshold of statistical significance. Due to the explorative nature of this study, no adjustment for multiple testing was done. All analyses were carried out using the software R (Version 4.0.4, www.rproject.org).

\section{Results}

Ninety-eight male patients were eligible for statistical analysis. Patient allocation to the study groups is shown in Fig. 1. Patient characteristics, duration of surgery, and anaesthesia are listed in Table 1. Mean age was 64 years ( \pm 7 years), mean BMI $26.9 \mathrm{~kg} / \mathrm{m}^{2}\left( \pm 3.3 \mathrm{~kg} / \mathrm{m}^{2}\right)$, and mean PBW $72.6 \mathrm{~kg}( \pm 4.7 \mathrm{~kg})$. Mean duration of surgery was $160 \mathrm{~min}( \pm 36 \mathrm{~min}$ ) and mean duration of anaesthesia $228 \mathrm{~min}( \pm 40 \mathrm{~min})$. The four study groups did not differ with regard to any of the described parameters and the infused crystalloid volume in each group complied with the study protocol. 
Table 1

Baseline patient and surgical characteristics $(n=98)$

\begin{tabular}{|c|c|c|c|c|c|}
\hline & $\begin{array}{l}\text { PEEP }_{5} \text { and liberal } \\
\text { volume }(n=23)\end{array}$ & $\begin{array}{l}\mathrm{PEEP}_{5} \text { and } \\
\text { restrictive } \\
\text { volume } \\
(\mathrm{n}=27)\end{array}$ & $\begin{array}{l}\text { PEEP }_{\text {IND }} \text { and } \\
\text { liberal volume } \\
(n=24)\end{array}$ & $\begin{array}{l}\text { PEEP }_{\text {IND }} \text { and } \\
\text { restrictive } \\
\text { volume } \\
(n=24)\end{array}$ & $\begin{array}{l}\mathrm{P}- \\
\text { value }\end{array}$ \\
\hline Age (years) & $64.57( \pm 5.72)$ & $62.81( \pm 6.94)$ & $63.96( \pm 6.68)$ & $63.5( \pm 8.67)$ & 0.846 \\
\hline Height (cm) & $177.48( \pm 5.72)$ & $178.44( \pm 4.3)$ & $\begin{array}{l}175.38( \pm \\
5.29)\end{array}$ & $177.29( \pm 4.98)$ & 0.193 \\
\hline Weight (kg) & $89.15( \pm 14.96)$ & $84.5( \pm 9.87)$ & $82.23( \pm 9.54)$ & $82.29( \pm 11.29)$ & 0.143 \\
\hline BMI $\left(\mathrm{kg} / \mathrm{m}^{2}\right)$ & $28.17( \pm 3.48)$ & $26.57( \pm 3.24)$ & $26.8( \pm 3.39)$ & $26.13( \pm 3.04)$ & 0.177 \\
\hline PBW (kg) & $72.82( \pm 5.2)$ & $73.7( \pm 3.91)$ & $70.91( \pm 4.81)$ & $72.65( \pm 4.53)$ & 0.193 \\
\hline $\begin{array}{l}\text { Volume / PBW } \\
\text { (ml/kg) }\end{array}$ & $30.8( \pm 2.3)$ & $15.5( \pm 2.62)$ & $29.6( \pm 2.8)$ & $15.8( \pm 2.82)$ & $\begin{array}{l}<.001 \\
0.00\end{array}$ \\
\hline $\begin{array}{l}\text { Duration of } \\
\text { surgery (min) }\end{array}$ & $165( \pm 35)$ & $155( \pm 33)$ & $165( \pm 43)$ & $153( \pm 35)$ & 0.532 \\
\hline $\begin{array}{l}\text { Duration of } \\
\text { anesthesia } \\
\text { (min) }\end{array}$ & $228( \pm 41)$ & $221( \pm 39)$ & $235( \pm 43)$ & $228( \pm 39)$ & 0.703 \\
\hline \multicolumn{6}{|c|}{ 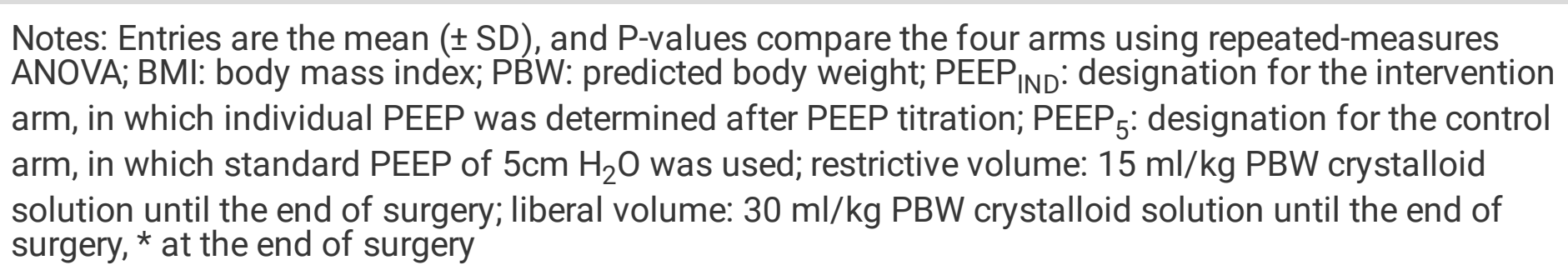 } \\
\hline
\end{tabular}

\section{Postoperative pulmonary function, body weight, and brain natriuretic peptide}

The results of pre- and postoperative spirometry are shown in Table 2 and Table S1. In the recovery room, no significant differences in spirometric parameters were observed between the study groups. On the first and second postoperative day, patients with an individualized higher PEEP level showed a significantly higher mean Tiffeneau index than patients with a standard PEEP level (1st day: $77.5 \%$ vs $73.6 \%, P=$ 0.014; 2 nd day: $76.5 \%$ vs $72.7 \%, P=0.021$ ) and higher $F F_{25}-75$ (1 st day: 2.41 vs $1.95 \mathrm{l} / \mathrm{sec}, \mathrm{P}=0.009$; 2nd day: 2.45 vs $2.07 \mathrm{l} / \mathrm{sec}, \mathrm{P}=0.033$ ). Postoperative spirometric parameters were not influenced by restrictive or liberal crystalloid infusion in either of the two PEEP groups. 
Table 2

Spirometric parameters compared between PEEP groups at four time points: on the day before surgery, in the recovery room, and on the first and second postoperative day $(n=98)$

\begin{tabular}{|c|c|c|c|c|}
\hline Parameters & Time point & $\begin{array}{l}\text { PEEP }_{5} \\
(n=50)\end{array}$ & $\begin{array}{l}\text { PEEP }_{\text {IND }} \\
(n=48)\end{array}$ & P-value \\
\hline \multirow{4}{*}{$\begin{array}{l}\text { VC } \\
\text { (liter) }\end{array}$} & preoperative & $4.46( \pm 0.9)$ & $4.39( \pm 0.7)$ & 0.610 \\
\hline & recovery room & $3.32( \pm 0.9)$ & $3.31( \pm 0.8)$ & 0.993 \\
\hline & postop day 1 & $3.48( \pm 0.8)$ & $3.52( \pm 0.7)$ & 0.843 \\
\hline & postop day 2 & $3.83( \pm 0.7)$ & $3.78( \pm 0.7)$ & 0.726 \\
\hline \multirow{4}{*}{$\begin{array}{l}\text { FVC } \\
\text { (liter) }\end{array}$} & preoperative & $4.32( \pm 0.8)$ & $4.16( \pm 0.6)$ & 0.294 \\
\hline & recovery room & $3.45( \pm 0.9)$ & $3.45( \pm 0.8)$ & 0.986 \\
\hline & postop day 1 & $3.46( \pm 0.8)$ & $3.50( \pm 0.7)$ & 0.803 \\
\hline & postop day 2 & $3.79( \pm 0.8)$ & $3.70( \pm 0.7)$ & 0.532 \\
\hline \multirow{4}{*}{$\begin{array}{l}\text { FEV1 } \\
\text { (liter) }\end{array}$} & preoperative & $3.16( \pm 0.6)$ & $3.13( \pm 0.5)$ & 0.811 \\
\hline & recovery room & $2.42( \pm 0.6)$ & $2.49( \pm 0.6)$ & 0.600 \\
\hline & postop day 1 & $2.54( \pm 0.6)$ & $2.71( \pm 0.6)$ & 0.151 \\
\hline & postop day 2 & $2.74( \pm 0.6)$ & $2.83( \pm 0.6)$ & 0.457 \\
\hline \multirow{4}{*}{$\begin{array}{l}\mathrm{FEV}_{1} / \mathrm{FVC} \text { ratio } \\
\text { (Tiffenau index) } \\
(\%)\end{array}$} & preoperative & $73.4( \pm 7.6)$ & $75.8( \pm 8.1)$ & 0.224 \\
\hline & recovery room & $70.7( \pm 10.2)$ & $72.0( \pm 8.5)$ & 0.484 \\
\hline & postop day 1 & $73.6( \pm 8.8)$ & $77.6( \pm 6.6)$ & $0.014^{*}$ \\
\hline & postop day 2 & $72.7( \pm 9.3)$ & $76.5( \pm 6.1)$ & $0.021 *$ \\
\hline \multirow{4}{*}{$\begin{array}{l}\text { PEF } \\
\text { (liter/min) }\end{array}$} & preoperative & $471.1( \pm 114.3)$ & $455.2( \pm 120.1)$ & 0.502 \\
\hline & recovery room & $299.2( \pm$ 104.9) & $292.6( \pm 98.6)$ & 0.749 \\
\hline & postop day 1 & $361.6( \pm 115.4)$ & $381.0( \pm 99.9)$ & 0.377 \\
\hline & postop day 2 & $372.0( \pm 113.2)$ & $388.4( \pm 106.8)$ & 0.466 \\
\hline \multirow{2}{*}{$\begin{array}{l}\mathrm{FEF}_{25} \\
\text { (liter/sec) }\end{array}$} & preoperative & $6.35( \pm 1.8)$ & $6.42( \pm 1.9)$ & 0.849 \\
\hline & recovery room & $4.51( \pm 1.8)$ & $4.34( \pm 1.6)$ & 0.617 \\
\hline \multicolumn{5}{|c|}{ 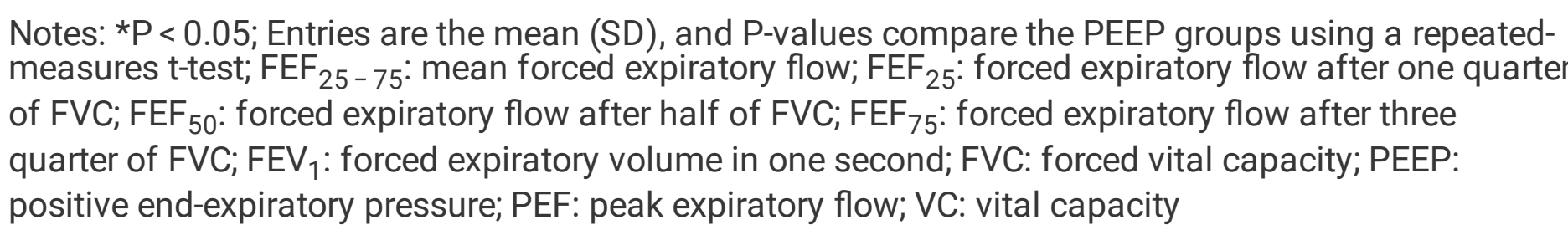 } \\
\hline
\end{tabular}




\begin{tabular}{|c|c|c|c|c|}
\hline Parameters & Time point & $\begin{array}{l}\text { PEEP }_{5} \\
(n=50)\end{array}$ & $\begin{array}{l}\text { PEEP }_{\text {IND }} \\
(n=48)\end{array}$ & P-value \\
\hline & postop day 1 & $4.99( \pm 1.9)$ & $5.68( \pm 1.6)$ & 0.057 \\
\hline & postop day 2 & $5.27( \pm 1.9)$ & $5.72( \pm 1.7)$ & 0.220 \\
\hline \multirow{4}{*}{$\begin{array}{l}\mathrm{FEF}_{50} \\
\text { (liter/sec) }\end{array}$} & preoperative & $3.39( \pm 1.3)$ & $3.77( \pm 1.4)$ & 0.172 \\
\hline & recovery room & $2.57( \pm 1.2)$ & $2.67( \pm 1.1)$ & 0.664 \\
\hline & postop day 1 & $2.73( \pm 1.1)$ & $3.36( \pm 1.1)$ & $0.005^{*}$ \\
\hline & postop day 2 & $2.88( \pm 1.1)$ & $3.49( \pm 1.4)$ & $0.018 *$ \\
\hline \multirow{4}{*}{$\begin{array}{l}\text { FEF }_{75} \\
\text { (liter/sec) }\end{array}$} & preoperative & $0.89( \pm 0.5)$ & $1.05( \pm 0.5)$ & 0.098 \\
\hline & recovery room & $0.72( \pm 0.4)$ & $0.85( \pm 0.4)$ & 0.119 \\
\hline & postop day 1 & $0.78( \pm 0.4)$ & $0.96( \pm 0.5)$ & 0.061 \\
\hline & postop day 2 & $0.81( \pm 0.5)$ & $0.92( \pm 0.4)$ & 0.227 \\
\hline \multirow{4}{*}{$\begin{array}{l}\mathrm{FEF}_{25-75} \\
\text { (liter/sec) }\end{array}$} & preoperative & $2.36( \pm 1.0)$ & $2.65( \pm 0.9)$ & 0.139 \\
\hline & recovery room & $1.80( \pm 0.8)$ & $1.98( \pm 0.8)$ & 0.273 \\
\hline & postop day 1 & $1.95( \pm 0.8)$ & $2.41( \pm 0.9)$ & $0.009 *$ \\
\hline & postop day 2 & $2.07( \pm 0.8)$ & $2.45( \pm 0.9)$ & $0.033^{*}$ \\
\hline \multicolumn{5}{|c|}{ 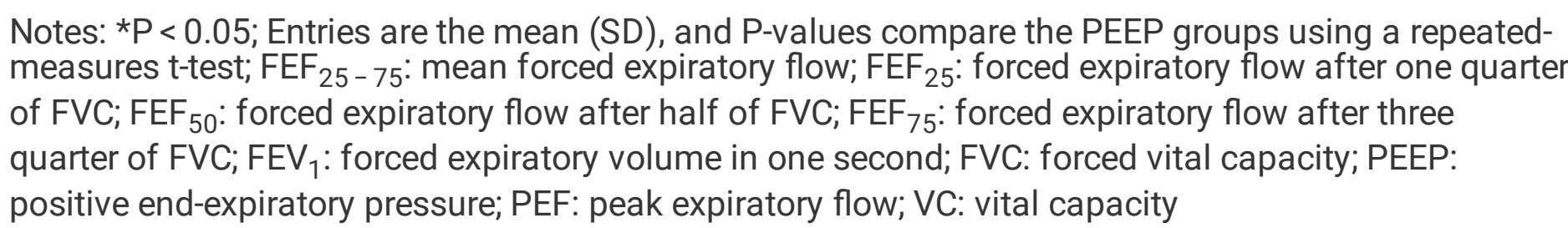 } \\
\hline
\end{tabular}

Preoperative values and postoperative changes in body weight and BNP levels are shown in Table S2. In each study group, mean body weight increased on the first postoperative day and decreased on the second postoperative day compared to preoperative values. Mean BNP level was preoperatively elevated in all study groups compared to normal values and increased on the first postoperative day. On the second postoperative day, mean BNP level had decreased below preoperative values in each study group. The study groups did not show any significant differences.

\section{Perioperative ventilation, haemodynamic and blood oxygenation}

The respiratory parameters of the four groups are depicted in Table 3 and Fig. 3. The mean PEEP was $15.5 \mathrm{cmH}_{2} \mathrm{O}\left( \pm 1.71 \mathrm{cmH}_{2} \mathrm{O}\right)$ in the individualized PEEP groups together. The individualized PEEP groups had significantly higher PIP and MP levels but significantly lower $P_{\text {driv }}$ levels at all measuring time points 
during RALP than the standard PEEP groups $\left(5 \mathrm{cmH}_{2} \mathrm{O}\right)$. Perioperative $\mathrm{P}_{\text {driv }}$ levels were lowest in the individualized PEEP group with restrictive crystalloid infusion. Dynamic LC and blood oxygenation measured by the P/F ratio and $\mathrm{SaO}_{2}$ were significantly higher in the individualized higher PEEP groups. 
Table 3

Mechanical ventilation, blood gas and haemodynamic parameters at five time points during RALP $(n=$ 98)

\begin{tabular}{|c|c|c|c|c|c|c|}
\hline Parameters & & $\begin{array}{l}\text { PEEP }_{5} \text { and } \\
\text { liberal } \\
\text { volume } \\
(n=23)\end{array}$ & $\begin{array}{l}\mathrm{PEEP}_{5} \text { and } \\
\text { restrictive } \\
\text { volume } \\
(n=27)\end{array}$ & $\begin{array}{l}\text { PEEP }_{\text {IND }} \text { and } \\
\text { liberal } \\
\text { volume } \\
(n=24)\end{array}$ & $\begin{array}{l}\text { PEEP }_{\text {IND }} \text { and } \\
\text { restrictive } \\
\text { volume } \\
(n=24)\end{array}$ & $\begin{array}{l}\text { P- } \\
\text { value }\end{array}$ \\
\hline \multirow{5}{*}{ 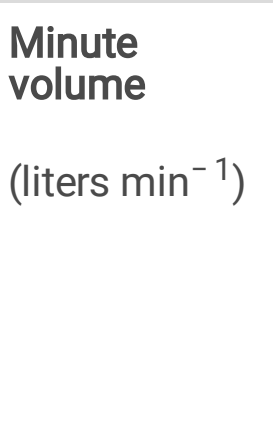 } & $\mathrm{T} 1$ & $7.3( \pm 0.9)$ & $6.9( \pm 0.7)$ & $6.7( \pm 0.8)$ & $6.8( \pm 1.1)$ & 0.055 \\
\hline & $\mathrm{T} 2$ & $8.2( \pm 1.1)$ & $8.1( \pm 0.9)$ & $7.7( \pm 1.0)$ & $8.0( \pm 1.1)$ & 0.323 \\
\hline & T3 & $8.6( \pm 1.2)$ & $8.1( \pm 1.0)$ & $8.2( \pm 1.1)$ & $8.3( \pm 1.6)$ & 0.574 \\
\hline & $\mathrm{T} 4$ & $8.7( \pm 2.0)$ & $8.2( \pm 1.0)$ & $8.1( \pm 1.1)$ & $8.1( \pm 1.2)$ & 0.370 \\
\hline & T5 & $8.6( \pm 2.1)$ & $8.4( \pm 1.3)$ & $8.1( \pm 1.6)$ & $8.3( \pm 1.6)$ & 0.735 \\
\hline \multirow{5}{*}{$\begin{array}{l}\text { Tidal volume } \\
\text { / PBW } \\
(\mathrm{ml} / \mathrm{kg})\end{array}$} & $\mathrm{T} 1$ & $8.3( \pm 0.9)$ & $7.7( \pm 0.5)$ & $8.0( \pm 0.7)$ & $7.7( \pm 0.8)$ & $0.010 *$ \\
\hline & $\mathrm{T} 2$ & $7.6( \pm 0.9)$ & $7.7( \pm 0.7)$ & $7.6( \pm 1.0)$ & $7.5( \pm 1.0)$ & 0.875 \\
\hline & T3 & $7.9( \pm 0.9)$ & $7.5( \pm 0.7)$ & $7.6( \pm 1.1)$ & $7.5( \pm 1.0)$ & 0.567 \\
\hline & $\mathrm{T} 4$ & $7.9( \pm 1.3)$ & $7.5( \pm 0.7)$ & $7.4( \pm 1.2)$ & $7.7( \pm 1.1)$ & 0.524 \\
\hline & T5 & $8.1( \pm 1.3)$ & $7.9( \pm 0.7)$ & $8.2( \pm 0.7)$ & $7.9( \pm 0.8)$ & 0.508 \\
\hline \multirow{5}{*}{$\begin{array}{l}\text { PEEP } \\
\left(\mathrm{cmH}_{2} \mathrm{O}\right)\end{array}$} & $\mathrm{T} 1$ & $5( \pm 0)$ & $5( \pm 0)$ & $5( \pm 0)$ & $5( \pm 0)$ & 0.357 \\
\hline & $\mathrm{T} 2$ & $5( \pm 0)$ & $5( \pm 0)$ & $15.2( \pm 1.5)$ & $15.6( \pm 2.0)$ & ¿ $0.001 *$ \\
\hline & T3 & $5( \pm 0)$ & $5( \pm 0)$ & $15.1( \pm 1.6)$ & $15.6( \pm 2.0)$ & <.001* \\
\hline & $\mathrm{T} 4$ & $5( \pm 0)$ & $5( \pm 0)$ & $15.1( \pm 1.7)$ & $15.6( \pm 2.0)$ & $<.001 *$ \\
\hline & T5 & $5( \pm 0)$ & $5( \pm 0)$ & $15.1( \pm 1.6)$ & $15.6( \pm 2.0)$ & <.001* \\
\hline PIP & $\mathrm{T} 1$ & $15.2( \pm 2.2)$ & $14.9( \pm 2.3)$ & $14.7( \pm 2.1)$ & $14.5( \pm 1.7)$ & 0.711 \\
\hline
\end{tabular}

$\left(\mathrm{cmH}_{2} \mathrm{O}\right)$

Notes: ${ }^{\mathrm{p}}<0.05$; Entries are the mean (SD), and $\mathrm{p}$-values compare the four arms using repeatedmeasures ANOVA; $\mathrm{PaCO}_{2}$ : partial pressure of carbon dioxide in arterial blood; PBW: predicted body weight; PEEP ${ }_{I N D}$ : individual PEEP was determined with titration; PEEP 5 : standard PEEP of $5 \mathrm{~cm} \mathrm{H}_{2} \mathrm{O}$ was used; restrictive volume: $15 \mathrm{ml} / \mathrm{kg}$ PBW crystalloid solution until the end of surgery; liberal volume: $30 \mathrm{ml} / \mathrm{kg}$ PBW crystalloid solution until the end of surgery; PIP: peak inspiratory pressure; T1: 5 min after intubation in supine position, T2: 30 min after start of capnoperitoneum and Trendelenburg position, T3: 60 min after start of capnoperitoneum and Trendelenburg position, T4: 90 min after start of capnoperitoneum and Trendelenburg position, T5: before extubation in supine position; \#calculated with the following formula: mechanical power $=0.098^{\star}$ tidal volume rate*(PIP-0,5*driving pressure) 


\begin{tabular}{|c|c|c|c|c|c|c|}
\hline \multirow[t]{5}{*}{ Parameters } & & $\begin{array}{l}\text { PEEP }_{5} \text { and } \\
\text { liberal } \\
\text { volume } \\
(n=23)\end{array}$ & $\begin{array}{l}\text { PEEP }_{5} \text { and } \\
\text { restrictive } \\
\text { volume } \\
(n=27)\end{array}$ & $\begin{array}{l}\text { PEEP }_{\text {IND }} \text { and } \\
\text { liberal } \\
\text { volume } \\
(n=24)\end{array}$ & $\begin{array}{l}\text { PEEP }_{\text {IND }} \text { and } \\
\text { restrictive } \\
\text { volume } \\
(n=24)\end{array}$ & $\begin{array}{l}\mathrm{P}- \\
\text { value }\end{array}$ \\
\hline & $\mathrm{T} 2$ & $26.4( \pm 4.0)$ & $24.9( \pm 5.4)$ & $28.8( \pm 3.1)$ & $29.2( \pm 2.9)$ & 0.001 * \\
\hline & T3 & $27.0( \pm 4.2)$ & $26.2( \pm 4.8)$ & $29.8( \pm 3.3)$ & $28.5( \pm 3.1)$ & 0.010 * \\
\hline & T4 & $28.0( \pm 4.5)$ & $26.6( \pm 3.5)$ & $30.1( \pm \pm 3.2)$ & $30.0( \pm 2.6)$ & 0.001 * \\
\hline & T5 & $17.4( \pm 1.8)$ & $16.5( \pm 2.2)$ & $24.3( \pm 2.2)$ & $25.2( \pm 2.5)$ & ¿. 001 * \\
\hline \multirow{5}{*}{$\begin{array}{l}\text { Driving } \\
\text { pressure } \\
\left(\mathrm{cmH}_{2} \mathrm{O}\right)\end{array}$} & $\mathrm{T} 1$ & $10.2( \pm 2.2)$ & $9.9( \pm 2.3)$ & $9.7( \pm 2.1)$ & $9.5( \pm 1.7)$ & 0.711 \\
\hline & T2 & $21.4( \pm 4.0)$ & $19.9( \pm 5.4)$ & $13.6( \pm 2.7)$ & $13.5( \pm 2.7)$ & <.001* \\
\hline & T3 & $22.0( \pm 4.2)$ & $21.2( \pm 4.8)$ & $14.7( \pm 3.5)$ & $12.9( \pm 2.8)$ & $<.001 *$ \\
\hline & T4 & $23.0( \pm 4.5)$ & $21.6( \pm 3.5)$ & $15.0( \pm 2.9)$ & $14.3( \pm 2.7)$ & $<.001 *$ \\
\hline & T5 & $12.4( \pm 1.8)$ & $11.5( \pm 2.2)$ & $9.2( \pm 1.4)$ & $9.6( \pm 1.8)$ & $5.001 *$ \\
\hline \multirow{5}{*}{$\begin{array}{l}\text { Dynamic lung } \\
\text { compliance } \\
\left(\mathrm{ml} \mathrm{cm} \mathrm{H} \mathrm{O}^{-}\right. \\
\left.{ }^{1}\right)\end{array}$} & $\mathrm{T} 1$ & $80.7( \pm 18.7)$ & $79.7( \pm 21.2)$ & $82.2( \pm 21.0)$ & $80.9( \pm 18.9)$ & 0.979 \\
\hline & T2 & $31.6( \pm 7.7)$ & $37.0( \pm 13.3)$ & $48.3( \pm 14.7)$ & $57.4( \pm 19.7)$ & <.001* \\
\hline & T3 & $31.5( \pm 7.8)$ & $33.9( \pm 9.9)$ & $45.6( \pm 13.8)$ & $55.3( \pm 13.4)$ & <. 001 * \\
\hline & T4 & $29.7( \pm 8.2)$ & $30.8( \pm 7.3)$ & $44.4( \pm 15.6)$ & $51.8( \pm 15.3)$ & $<.001 *$ \\
\hline & T5 & $67.5( \pm 28.4)$ & $70.5( \pm 20.3)$ & $91.9( \pm 31.8)$ & $89.1( \pm 14.3)$ & $\hat{0.001 *}$ \\
\hline $\begin{array}{l}\text { Mechanical } \\
\text { power" } \\
(\mathrm{J} / \mathrm{min})\end{array}$ & $\mathrm{T} 1$ & $10.0( \pm 1.9)$ & $9.1( \pm 1.4)$ & $9.1( \pm 1.4)$ & $9.0( \pm 1.9)$ & 0.099 \\
\hline \multicolumn{7}{|c|}{ 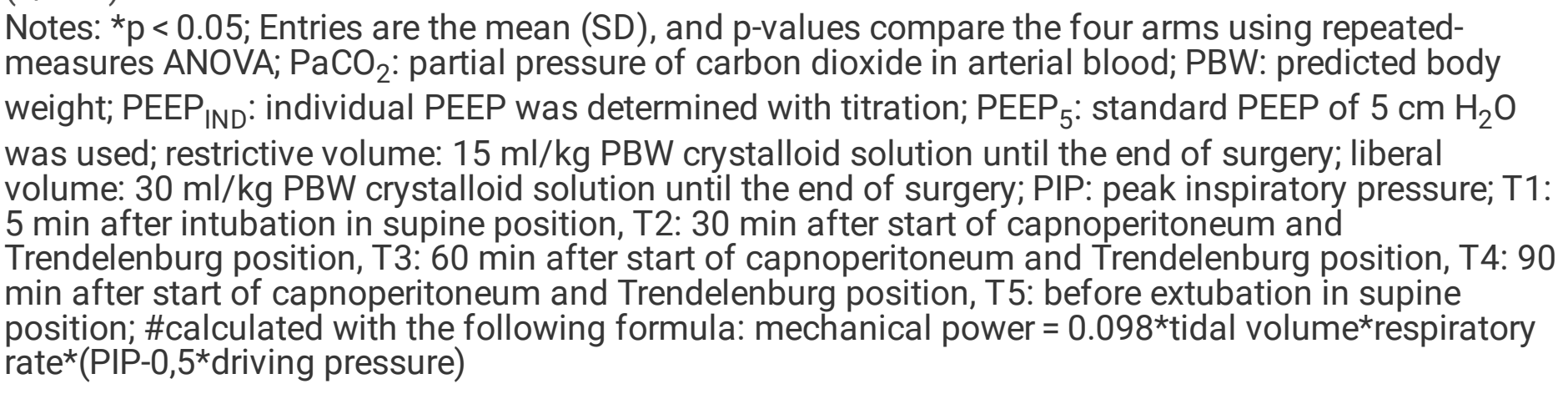 } \\
\hline
\end{tabular}




\begin{tabular}{|c|c|c|c|c|c|c|}
\hline \multicolumn{2}{|l|}{ Parameters } & $\begin{array}{l}\text { PEEP }_{5} \text { and } \\
\text { liberal } \\
\text { volume } \\
(n=23)\end{array}$ & $\begin{array}{l}\mathrm{PEEP}_{5} \text { and } \\
\text { restrictive } \\
\text { volume } \\
(\mathrm{n}=27)\end{array}$ & $\begin{array}{l}\text { PEEP }_{\text {IND }} \text { and } \\
\text { liberal } \\
\text { volume } \\
(n=24)\end{array}$ & $\begin{array}{l}\text { PEEP }_{\text {IND }} \text { and } \\
\text { restrictive } \\
\text { volume } \\
(n=24)\end{array}$ & $\begin{array}{l}\mathrm{P}- \\
\text { value }\end{array}$ \\
\hline \multirow{9}{*}{ pH } & $\mathrm{T} 2$ & $17.4( \pm 3.4)$ & $16.2( \pm 3.9)$ & $23.4( \pm 4.0)$ & $24.0( \pm 3.5)$ & ¿. $0.001^{\star}$ \\
\hline & T3 & $18.5( \pm 3.6)$ & $16.9( \pm 3.5)$ & $25.4( \pm 3.9)$ & $24.5( \pm 5.0)$ & <. $001 *$ \\
\hline & $\mathrm{T4}$ & $19.3( \pm 5.2)$ & $17.2( \pm 3.3)$ & $25.2( \pm 3.9)$ & $25.0( \pm 3.8)$ & <. $001 *$ \\
\hline & T5 & $13.1( \pm 3.6)$ & $12.0( \pm 2.1)$ & $22.1( \pm 4.9)$ & $23.0( \pm 5.5)$ & <.001* \\
\hline & $\mathrm{T} 1$ & $7.40( \pm 0.03)$ & $7.40( \pm 0.03)$ & $7.41( \pm 0.03)$ & $7.41( \pm 0.03)$ & 0.299 \\
\hline & $\mathrm{T} 2$ & $7.37( \pm 0.02)$ & $7.37( \pm 0.03)$ & $7.37( \pm 0.04)$ & $7.38( \pm 0.03)$ & 0.631 \\
\hline & T3 & $7.37( \pm 0.03)$ & $7.37( \pm 0.03)$ & $7.36( \pm 0.04)$ & $7.37( \pm 0.04)$ & 0.874 \\
\hline & $\mathrm{T4}$ & $7.36( \pm 0.04)$ & $7.36( \pm 0.04)$ & $7.35( \pm 0.05)$ & $7.35( \pm 0.04)$ & 0.980 \\
\hline & T5 & $7.35( \pm 0.04)$ & $7.34( \pm 0.04)$ & $7.34( \pm 0.04)$ & $7.34( \pm 0.04)$ & 0.848 \\
\hline \multirow{5}{*}{$\begin{array}{l}\mathrm{PaCO}_{2} \\
(\mathrm{mmHg})\end{array}$} & $\mathrm{T} 1$ & $39.0( \pm 3.5)$ & $37.9( \pm 3.5)$ & $37.5( \pm 3.1)$ & $37.5( \pm 3.4)$ & 0.406 \\
\hline & $\mathrm{T} 2$ & $40.7( \pm 2.9)$ & $39.6( \pm 3.1)$ & $41.0( \pm 4.0)$ & $39.9( \pm 3.4)$ & 0.406 \\
\hline & T3 & $41.7( \pm 3.9)$ & $40.1( \pm 3.6)$ & $41.5( \pm 4.2)$ & $40.8( \pm 4.2)$ & 0.462 \\
\hline & $\mathrm{T} 4$ & $42.5( \pm 5.6)$ & $41.2( \pm 3.1)$ & $42.3( \pm 4.3)$ & $42.4( \pm 4.9)$ & 0.713 \\
\hline & T5 & $42.5( \pm 5.5)$ & $42.6( \pm 4.3)$ & $41.9( \pm 4.7)$ & $42.0( \pm 5.7)$ & 0.957 \\
\hline \multirow{4}{*}{$\begin{array}{l}\mathrm{PaO}_{2} / \mathrm{FiO}_{2} \\
(\mathrm{mmHg})\end{array}$} & $\mathrm{T} 1$ & $420( \pm 93)$ & $402( \pm 115)$ & $428( \pm 112)$ & $395( \pm 118)$ & 0.715 \\
\hline & T2 & $329( \pm 100)$ & $334( \pm 94)$ & $408( \pm 87)$ & $425( \pm 86)$ & $<.001^{*}$ \\
\hline & T3 & $316( \pm 103)$ & $320( \pm 103)$ & $398( \pm 92)$ & $397( \pm 90)$ & $0.002^{\star}$ \\
\hline & $\mathrm{T} 4$ & $315( \pm 99)$ & $315( \pm 87)$ & $382( \pm 93)$ & $380( \pm 82)$ & $0.007^{\star}$ \\
\hline \multicolumn{7}{|c|}{ 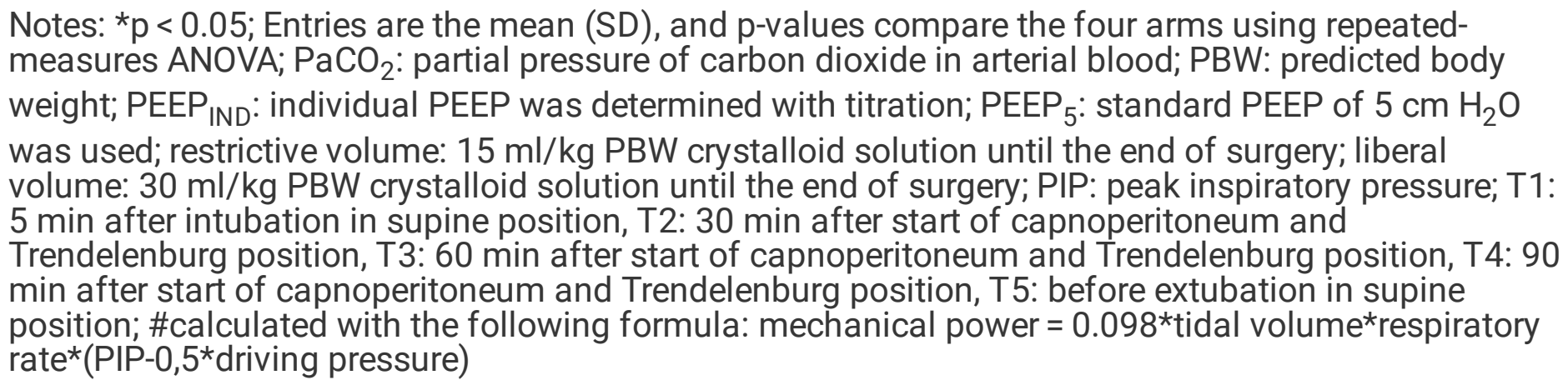 } \\
\hline
\end{tabular}




\begin{tabular}{|c|c|c|c|c|c|c|}
\hline \multirow[t]{2}{*}{ Parameters } & & $\begin{array}{l}\text { PEEP }_{5} \text { and } \\
\text { liberal } \\
\text { volume } \\
(n=23)\end{array}$ & $\begin{array}{l}\text { PEEP }_{5} \text { and } \\
\text { restrictive } \\
\text { volume } \\
(n=27)\end{array}$ & $\begin{array}{l}\text { PEEP }_{\text {IND }} \text { and } \\
\text { liberal } \\
\text { volume } \\
(n=24)\end{array}$ & $\begin{array}{l}\text { PEEP }_{\text {IND }} \text { and } \\
\text { restrictive } \\
\text { volume } \\
(n=24)\end{array}$ & $\begin{array}{l}\mathrm{P}- \\
\text { value }\end{array}$ \\
\hline & T5 & $339( \pm 90)$ & $336( \pm 83)$ & $395( \pm 83)$ & $413( \pm 76)$ & $0.002^{*}$ \\
\hline \multirow{5}{*}{$\begin{array}{l}\text { Mean arterial } \\
\text { pressure } \\
(\mathrm{mmHg})\end{array}$} & $\mathrm{T} 1$ & $74.5( \pm 11.0)$ & $78.6( \pm 12.4)$ & $77.4( \pm 14.5)$ & $79.1( \pm 20.7)$ & 0.726 \\
\hline & $\mathrm{T} 2$ & $\begin{array}{l}103.4( \pm \\
10.7)\end{array}$ & $101.2( \pm 10.8)$ & $104.3( \pm 15.1)$ & $101.1( \pm 10.8)$ & 0.728 \\
\hline & T3 & $99.0( \pm 11.3)$ & $96.6( \pm 11.9)$ & $97.4( \pm 11.9)$ & $96.8( \pm 13.7)$ & 0.900 \\
\hline & $\mathrm{T} 4$ & $96.3( \pm 11.0)$ & $89.1( \pm 10.0)$ & $94.0( \pm 11.8)$ & $87.7( \pm 11.8)$ & $0.028^{*}$ \\
\hline & T5 & $82.5( \pm 10.8)$ & $78.9( \pm 10.6)$ & $74.6( \pm 8.1)$ & $78.8( \pm 11.2)$ & 0.077 \\
\hline \multirow{5}{*}{$\begin{array}{l}\text { Noradrenaline } \\
(\mathrm{mg} / \mathrm{h})\end{array}$} & $\mathrm{T} 1$ & $0.13( \pm 0.11)$ & $0.14( \pm 0.08)$ & $0.15( \pm 0.09)$ & $0.13( \pm 0.15)$ & 0.897 \\
\hline & $\mathrm{T} 2$ & $0( \pm 0)$ & $0.01( \pm 0.04)$ & $0.01( \pm 0.03)$ & $0.01( \pm 0.04)$ & 0.601 \\
\hline & T3 & $0( \pm 0)$ & $0( \pm 0.02)$ & $0.01( \pm 0.02)$ & $0.02( \pm 0.04)$ & 0.105 \\
\hline & $\mathrm{T4}$ & $0( \pm 0)$ & $0.01( \pm 0.03)$ & $0( \pm 0.02)$ & $0.01( \pm 0.05)$ & 0.327 \\
\hline & T5 & $0.16( \pm 0.11)$ & $0.21( \pm 0.13)$ & $0.23( \pm 0.13)$ & $0.29( \pm 0.17)$ & $0.010 *$ \\
\hline \multicolumn{7}{|c|}{ 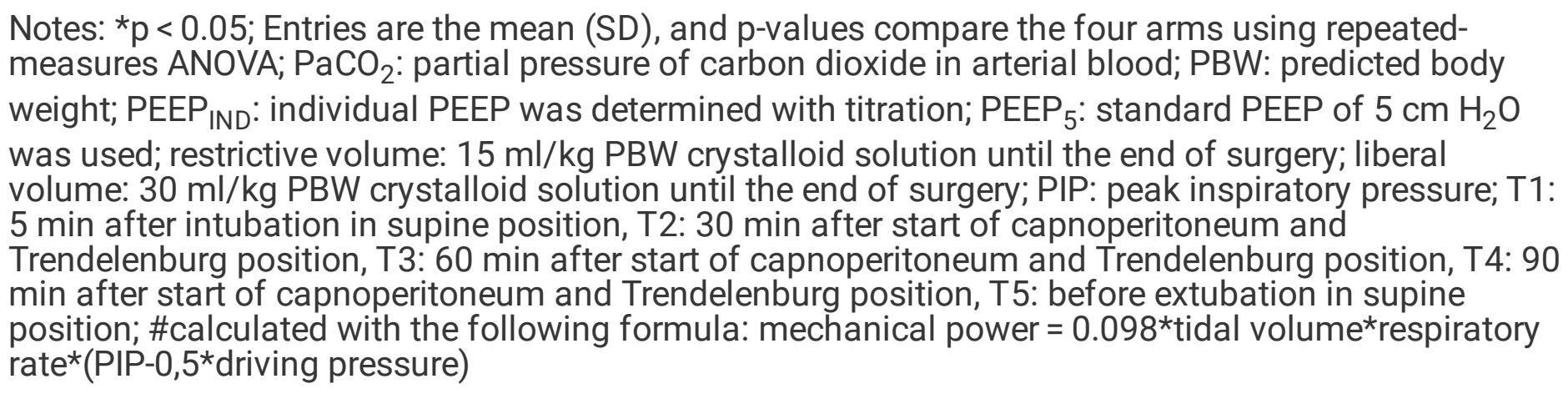 } \\
\hline
\end{tabular}

The two different crystalloid infusion groups did not differ in PIP, MP, PEEP, and Priv $_{\text {div }}$ levels, dynamic LC, Horovitz index, and $\mathrm{SaO}_{2}$ during RALP; only at T4 were the MAP values significantly lower in the group receiving restrictive crystalloid infusion ( 88.5 vs $95.1 \mathrm{mmHg}, \mathrm{P}=0.028$ ).

\section{Discussion}

This study including 98 patients analysed the influence of different perioperative PEEP levels and crystalloid infusion on postoperative pulmonary function up to 48 hours after RALP. The main findings were: 1) pulmonary function was significantly increased in the group of patients with perioperatively higher PEEP levels up to the second postoperative day; 2) neither restrictive nor liberal crystalloid infusion during surgery had a significant influence on postoperative pulmonary function. Secondary findings were: 
1) the application of individual higher PEEP values significantly increased MP, perioperative oxygenation $\left(\mathrm{PaO}_{2} / \mathrm{FiO}_{2}\right.$ ratio), $\mathrm{LC}$ and significantly decreased driving pressure. 2) The study groups did not significantly differ in pre-and postoperative BNP levels and body weight.

\section{Postoperative pulmonary function, body weight, and brain natriuretic peptide}

The combination of STP and capnoperitoneum for two to three hours may cause oedema of the upper airway and reduce pulmonary compliance $[4,20]$. In our study, all spirometric parameters were decreased after RALP compared to preoperative measurements and showed the lowest values in the recovery room. Kilic and colleagues investigated the effects of STP for RALP on the intra- and extrathoracic airways in patients with and without COPD. In patients without COPD, VC and FEV ${ }_{1}$ were reduced after RALP but recovered within 5 days, whereas in patients with COPD, the alteration lasted more than 5 days [21]. The patients of our study with preoperative normal lung function showed significantly better Tiffeneau Index and higher $\mathrm{FEF}_{25-75}$ values in the group with higher PEEP values. While decreased Tiffenau values are a sign of increased bronchoconstriction in the larger airways, decreased $\mathrm{FEF}_{25-75}$ values represent higher flow resistance of the small airways of the lung. This could be a result of the increased capillary leakage during STP leading to increased flow resistances and that high individual PEEP may prevent the influx of fluid into the interstitial lung space.

In our study, the volume of administered crystalloids did not influence postoperative pulmonary function. No previous study had investigated the impact of different fluid strategies on intra- and postoperative pulmonary function during RALP. Piegeler et al. evaluated the influence of intraoperative fluid administration on postoperative complications (urologic complications and length of hospitalization) in 182 patients undergoing RALP. High amounts of crystalloids were associated with an increased incidence of anastomotic leakages, especially in older patients, but had no effect on the length of hospitalization [22].

Measuring circulating levels of BNP is a diagnostic aid in identifying patients with elevated ventricular filling pressure who are likely to develop symptoms of heart failure [23, 24]. STP and capnoperitoneum during RALP caused 2-to 3-fold increases in cardiac preload and may have resulted in acute heart failure $[20,25,26]$. Several previous studies have described perioperative cardiac ischemia during and after RALP $[27,28]$. In our study, no postoperative cardiac complications were observed. Mean BNP levels and body weight increased on the first postoperative day but normalized on the second postoperative day, independent of PEEP and volume administrations. To the best of our knowledge, no previous study investigated changes in BNP and bodyweight levels in patients undergoing RALP.

\section{Perioperative ventilation and blood oxygenation}

In this study, individualized higher PEEP levels resulted in better blood oxygenation $\left(\mathrm{SaO}_{2}\right.$, Horovitz index) and lung-protective ventilation (increased $L C$ and reduced $P_{\text {driv }}$ ) but resulted in a significantly higher 
calculated MP on the lungs, probably due to the application of significantly higher individual PEEP levels. The promising idea to combine several variables related to ventilator-induced lung injury (VILI) in the equation of MP [29] still has several limitations especially proper modeling of PEEP and has some qualitative disagreements with clinical data on VILI [30] as we saw in our study. High MP to lungs as seen in our high PEEP group may probably be of harmful character on one hand, on the other hand, we observed a decrease in $\mathrm{P}_{\text {driv }}$ and better oxygenation, $\mathrm{LC}$, and postoperative spirometric lung function, though it shouldn't be overestimated. Nestler et al. analysed obese patients undergoing elective laparoscopic bariatric surgery in general anaesthesia and found that higher PEEP levels improved blood oxygenation and significantly reduced $\mathrm{P}_{\text {driv, }}$, but these differences had not persisted after extubation [31]. Inadequately low PEEP values during laparoscopic surgery (in STP) favor the development of atelectasis and decreases pulmonary ventilation and oxygenation. Two current studies using electrical impedance tomography during RALP shows that a PEEP with 14 to $15 \mathrm{cmH}_{2} \mathrm{O}$ improves oxygenation in non-obese individuals [32, 33].

$\mathrm{V}_{\mathrm{T}}$ changes during robot-assisted laparoscopic surgery result in fluid shifts detected by pulse pressure variation and stroke volume variation [34]. Otherwise, passive leg raise does not predict fluid responsiveness in patients with IAP $>16 \mathrm{mmHg}$ [35]. Neither restrictive nor liberal crystalloid management influenced perioperative ventilation and oxygenation in our study. One possible explanation for this result is that abdominal laparoscopic surgery may potentially influence dynamic variables through direct mechanical effects and the autonomic nervous system, thus confounding the effects of different fluid challenges. Furthermore, capnoperitoneum probably affects the volume mobilized by head-down tilts.

\section{Strengths and limitations}

The strengths of the present study are its prospective randomized single-blinded design and the measurement of pulmonary function up to 48 hours after RALP in a large number of patients. Moreover, the study was performed only by a few doctors within a very short study time. This allowed a very strict standardization of the study process and accurately performed measurements. Taking into account the demanding requirements of mechanical ventilation during STP, we add MP calculations during laparoscopic surgery to the existing body of knowledge for the first time.

Nevertheless spirometry is a measurement method that is strongly dependent on the patient's cooperation. Due to the small sample size in the subgroups the effects of liberal or restrictive crystalloids no statement can be made regarding this issue.

\section{Conclusion}

Individualized higher PEEP levels during RALP improved blood oxygenation and driving pressure, but increased MP, and lead to an improved postoperative pulmonary function up to 48 hours after surgery. Restrictive crystalloid infusion during RALP seems to have no effect on peri- and postoperative oxygenation and pulmonary function. 


\section{Declarations}

\section{List of abbreviations}

$\begin{array}{ll}\text { BNP } & \text { brain natriuretic peptide } \\ \text { BMI } & \text { body mass index } \\ \text { COPD } & \text { chronic obstructive pulmonary disease } \\ \text { IAP } & \text { intra-abdominal pressure } \\ \text { LC } & \text { lung compliance } \\ \text { MAP } & \text { mean arterial blood pressure } \\ \text { MP } & \text { mechanical power } \\ \text { PBW } & \text { predicted body weight } \\ \text { Pdriv } & \text { driving pressure } \\ \text { PEEP } & \text { positive end-expiratory pressure } \\ \text { PIP } & \text { peak inspiratory pressure } \\ \text { RALP } & \text { robotic-assisted laparoscopic prostatectomy } \\ \text { RR } & \text { respiratory rate } \\ \text { SD } & \text { standard deviation } \\ \text { STP } & \text { steep Trendelenburg position } \\ \text { VILI } & \text { ventilator-induced lung injury } \\ \text { V tidal volume }\end{array}$

Ethics approval and consent to participate: This prospective, randomized, single-centre, and singleblinded pilot-study was approved by the local institutional review board (Protocol no. 18-1224-101, approved on 12 December 2018) and registered with the German Clinical Trials Register (DRKS00016887, prospectively registered on 7 March 2019). Informed consent was obtained from 104 patients scheduled for elective prostatectomy at the Department of Urology at the Caritas St. Josef University Medical Centre Regensburg 
Consent for publication: Not applicable

Availability of data and materials: The datasets supporting the conclusions of this article are included within the article and its additional files.

Competing interests: The authors declare no competing interests.

Funding: The study did not receive any extramural funding.

Authors' contributions: SB: Idea for the study, ethics approval, study design, data collection and analysis, drafting of the manuscript, and review of the manuscript for important intellectual content; $\mathrm{AH}$ : patient recruitment, data collection and interpretation, and review of the manuscript for important intellectual content; VG: patient recruitment, data collection and interpretation, and review of the manuscript for important intellectual content; TS: data collection and interpretation and review of the manuscript for important intellectual content; FZ: data analysis and review of the manuscript for important intellectual content; ML: study design, data interpretation, review of the manuscript for important intellectual content; MB: data collection and interpretation and review of the manuscript for important intellectual content; MP: study design, patient recruitment, data collection and interpretation, and review of the manuscript for important intellectual content; All authors have read and approved the manuscript.

Acknowledgements: We are grateful to Monika Schoell for the linguistic revision of the manuscript.

\section{References}

1. Tewari A, Sooriakumaran P, Bloch DA, Seshadri-Kreaden U, Hebert AE, Wiklund P. Positive Surgical Margin and Perioperative Complication Rates of Primary Surgical Treatments for Prostate Cancer: A Systematic Review and Meta-Analysis Comparing Retropubic, Laparoscopic, and Robotic Prostatectomy. Eur Urol. 2012;62:1-15. doi:10.1016/j.eururo.2012.02.029.

2. Novara G, Ficarra V, Rosen RC, Artibani W, Costello A, Eastham JA, et al. Systematic review and metaanalysis of perioperative outcomes and complications after robot-assisted radical prostatectomy. Eur Urol. 2012;62:431-52. doi:10.1016/j.eururo.2012.05.044.

3. Ficarra V, Novara G, Artibani W, Cestari A, Galfano A, Graefen M, et al. Retropubic, laparoscopic, and robot-assisted radical prostatectomy: A systematic review and cumulative analysis of comparative studies. Eur Urol. 2009;55:1037-63. doi:10.1016/j.eururo.2009.01.036.

4. Danic MJ, Chow M, Alexander G, Bhandari A, Menon M, Brown M. Anesthesia considerations for robotic-assisted laparoscopic prostatectomy: A review of 1,500 cases. J Robot Surg. 2007;1:119-23. doi:10.1007/s11701-007-0024-z. 
5. Gainsburg DM. Anesthetic concerns for robotic-assisted laparoscopic radical prostatectomy. Minerva Anestesiol. 2012;78:596-604.

6. SCOTT HARRIS R, HESS DR, VENEGAS JG. An Objective Analysis of the Pressure-Volume Curve in the Acute Respiratory Distress Syndrome. Am J Respir Crit Care Med. 2000;161:432-9. doi:10.1164/ajrccm.161.2.9901061.

7. Pelosi P, Rocco PRM, Gama de Abreu M. Close down the lungs and keep them resting to minimize ventilator-induced lung injury. Crit Care. 2018;22:72. doi:10.1186/s13054-018-1991-3.

8. Amato MBP, Meade MO, Slutsky AS, Brochard L, Costa ELV, Schoenfeld DA, et al. Driving Pressure and Survival in the Acute Respiratory Distress Syndrome. N Engl J Med. 2015;372:747-55. doi:10.1056/NEJMsa1410639.

9. Serpa Neto A, Cardoso SO, Manetta JA, Pereira VGM, Espósito DC, Pasqualucci MdOP, et al. Association between use of lung-protective ventilation with lower tidal volumes and clinical outcomes among patients without acute respiratory distress syndrome: A meta-analysis. JAMA. 2012;308:1651-9. doi:10.1001/jama.2012.13730.

10. Stewart RM, Park PK, Hunt JP, McIntyre RC, McCarthy J, Zarzabal LA, Michalek JE. Less Is More: Improved Outcomes in Surgical Patients with Conservative Fluid Administration and Central Venous Catheter Monitoring. Journal of the American College of Surgeons. 2009;208:725-35. doi:10.1016/j.jamcollsurg.2009.01.026.

11. Wiedemann HP, Wheeler AP, Bernard GR, Thompson BT, Hayden D, deBoisblanc B, et al. Comparison of two fluid-management strategies in acute lung injury. N Engl J Med. 2006;354:2564-75. doi:10.1056/NEJMoa062200.

12. Vignon P, Evrard B, Asfar P, Busana M, Calfee CS, Coppola S, et al. Fluid administration and monitoring in ARDS: Which management? Intensive Care Med. 2020;46:2252-64. doi:10.1007/s00134-020-06310-0.

13. Brandstrup B, Tønnesen H, Beier-Holgersen R, Hjorts $\varnothing$ E, Ørding H, Lindorff-Larsen K, et al. Effects of intravenous fluid restriction on postoperative complications: Comparison of two perioperative fluid regimens: a randomized assessor-blinded multicenter trial. Annals of surgery 2003. doi:10.1097/01.sla.0000094387.50865.23.

14. Myles PS, Bellomo R, Corcoran T, Forbes A, Peyton P, Story D, et al. Restrictive versus Liberal Fluid Therapy for Major Abdominal Surgery. N Engl J Med. 2018;378:2263-74. doi:10.1056/NEJMoa1801601.

15. Deane AM, Reid DA, Tobin AE. Predicted body weight during mechanical ventilation: Using arm demispan to aid clinical assessment. Crit Care Resusc. 2008;10:14.

16. Serpa Neto A, Deliberato RO, Johnson AEW, Bos LD, Amorim P, Pereira SM, et al. Mechanical power of ventilation is associated with mortality in critically ill patients: An analysis of patients in two observational cohorts. Intensive Care Med. 2018;44:1914-22. doi:10.1007/s00134-018-5375-6.

17. Gattinoni L, Tonetti T, Quintel M. Intensive care medicine in 2050: Ventilator-induced lung injury. Intensive Care Med. 2018;44:76-8. doi:10.1007/s00134-017-4770-8. 
18. Ponikowski P, Voors AA, Anker SD, Bueno H, Cleland JGF, Coats AJS, et al. 2016 ESC Guidelines for the diagnosis and treatment of acute and chronic heart failure: The Task Force for the diagnosis and treatment of acute and chronic heart failure of the European Society of Cardiology (ESC)Developed with the special contribution of the Heart Failure Association (HFA) of the ESC. Eur Heart J. 2016;37:2129-200. doi:10.1093/eurheartj/ehw128.

19. Whitehead AL, Julious SA, Cooper CL, Campbell MJ. Estimating the sample size for a pilot randomised trial to minimise the overall trial sample size for the external pilot and main trial for a continuous outcome variable. Stat Methods Med Res. 2016;25:1057-73. doi:10.1177/0962280215588241.

20. Kalmar AF, Foubert L, Hendrickx JFA, Mottrie A, Absalom A, Mortier EP, Struys MMRF. Influence of steep Trendelenburg position and $\mathrm{CO}(2)$ pneumoperitoneum on cardiovascular, cerebrovascular, and respiratory homeostasis during robotic prostatectomy. Br J Anaesth. 2010;104:433-9. doi:10.1093/bja/aeq018.

21. Kilic OF, Börgers A, Köhne W, Musch M, Kröpfl D, Groeben H. Effects of steep Trendelenburg position for robotic-assisted prostatectomies on intra- and extrathoracic airways in patients with or without chronic obstructive pulmonary disease. Br J Anaesth. 2015;114:70-6. doi:10.1093/bja/aeu322.

22. Piegeler T, Dreessen P, Graber SM, Haile SR, Schmid DM, Beck-Schimmer B. Impact of intraoperative fluid administration on outcome in patients undergoing robotic-assisted laparoscopic prostatectomy-a retrospective analysis. BMC Anesthesiol. 2014;14:61. doi:10.1186/1471-2253-1461.

23. Cowie MR, Struthers AD, Wood DA, Coats AJ, Thompson SG, Poole-Wilson PA, Sutton GC. Value of natriuretic peptides in assessment of patients with possible new heart failure in primary care. Lancet. 1997;350:1349-53. doi:10.1016/S0140-6736(97)06031-5.

24. Seino Y, Ogawa A, Yamashita T, Fukushima M, Ogata K-i, Fukumoto H, Takano T. Application of NTproBNP and BNP measurements in cardiac care: A more discerning marker for the detection and evaluation of heart failure. Eur J Heart Fail. 2004;6:295-300. doi:10.1016/j.ejheart.2003.12.009.

25. Lestar M, Gunnarsson L, Lagerstrand L, Wiklund P, Odeberg-Wernerman S. Hemodynamic perturbations during robot-assisted laparoscopic radical prostatectomy in $45^{\circ}$ Trendelenburg position. Anesth Analg. 2011;113:1069-75. doi:10.1213/ANE.0b013e3182075d1f.

26. Pawlik MT, Prasser C, Zeman F, Harth M, Burger M, Denzinger S, Blecha S. Pronounced haemodynamic changes during and after robotic-assisted laparoscopic prostatectomy: A prospective observational study. BMJ Open. 2020;10:e038045. doi:10.1136/bmjopen-2020-038045.

27. Blecha S, Harth M, Schlachetzki F, Zeman F, Blecha C, Flora P, et al. Changes in intraocular pressure and optic nerve sheath diameter in patients undergoing robotic-assisted laparoscopic prostatectomy in steep $45^{\circ}$ Trendelenburg position. BMC Anesthesiol. 2017;17:40. doi:10.1186/s12871-017-0333-3.

28. Ferroni MC, Abaza R. Feasibility of robot-assisted prostatectomy performed at ultra-low pneumoperitoneum pressure of $6 \mathrm{mmHg}$ and comparison of clinical outcomes vs standard pressure of 15 mmHg. BJU Int. 2019;124:308-13. doi:10.1111/bju.14682. 
29. Gattinoni L, Tonetti T, Cressoni M, Cadringher P, Herrmann P, Moerer O, et al. Ventilator-related causes of lung injury: The mechanical power. Intensive Care Med. 2016;42:1567-75. doi:10.1007/s00134016-4505-2.

30. Huhle R, Serpa Neto A, Schultz MJ, Gama de Abreu M. Is mechanical power the final word on ventilator-induced lung injury?-no. Ann Transl Med. 2018;6:394. doi:10.21037/atm.2018.09.65.

31. Nestler C, Simon P, Petroff D, Hammermüller S, Kamrath D, Wolf $S$, et al. Individualized positive endexpiratory pressure in obese patients during general anaesthesia: A randomized controlled clinical trial using electrical impedance tomography. Br J Anaesth. 2017;119:1194-205. doi:10.1093/bja/aex192.

32. Girrbach F, Petroff D, Schulz S, Hempel G, Lange M, Klotz C, et al. Individualised positive endexpiratory pressure guided by electrical impedance tomography for robot-assisted laparoscopic radical prostatectomy: A prospective, randomised controlled clinical trial. $\mathrm{Br} J$ Anaesth 2020. doi:10.1016/j.bja.2020.05.041.

33. Shono A, Katayama N, Fujihara T, Böhm SH, Waldmann AD, Ugata K, et al. Positive End-expiratory Pressure and Distribution of Ventilation in Pneumoperitoneum Combined with Steep Trendelenburg Position. Anesthesiology. 2020;132:476-90. doi:10.1097/ALN.0000000000003062.

34. Jun J-H, Chung RK, Baik HJ, Chung MH, Hyeon J-S, Lee Y-G, Park S-H. The tidal volume challenge improves the reliability of dynamic preload indices during robot-assisted laparoscopic surgery in the Trendelenburg position with lung-protective ventilation. BMC Anesthesiol. 2019;19:142. doi:10.1186/s12871-019-0807-6.

35. Mahjoub Y, Touzeau J, Airapetian N, Lorne E, Hijazi M, Zogheib E, et al. The passive leg-raising maneuver cannot accurately predict fluid responsiveness in patients with intra-abdominal hypertension. Crit Care Med. 2010;38:1824-9. doi:10.1097/CCM.0b013e3181eb3c21.

\section{Figures}




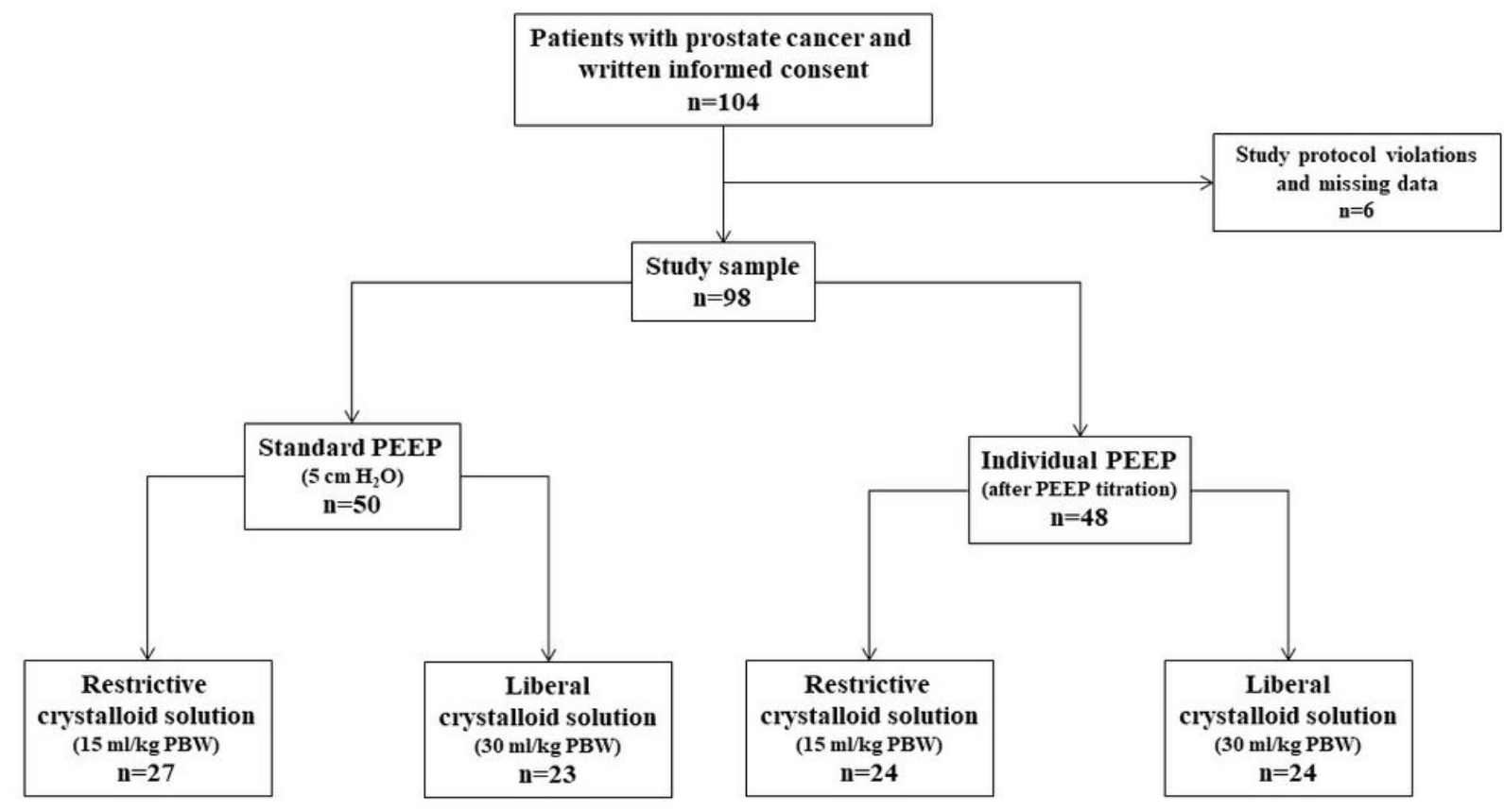

Figure 1

Patient flow chart of the study
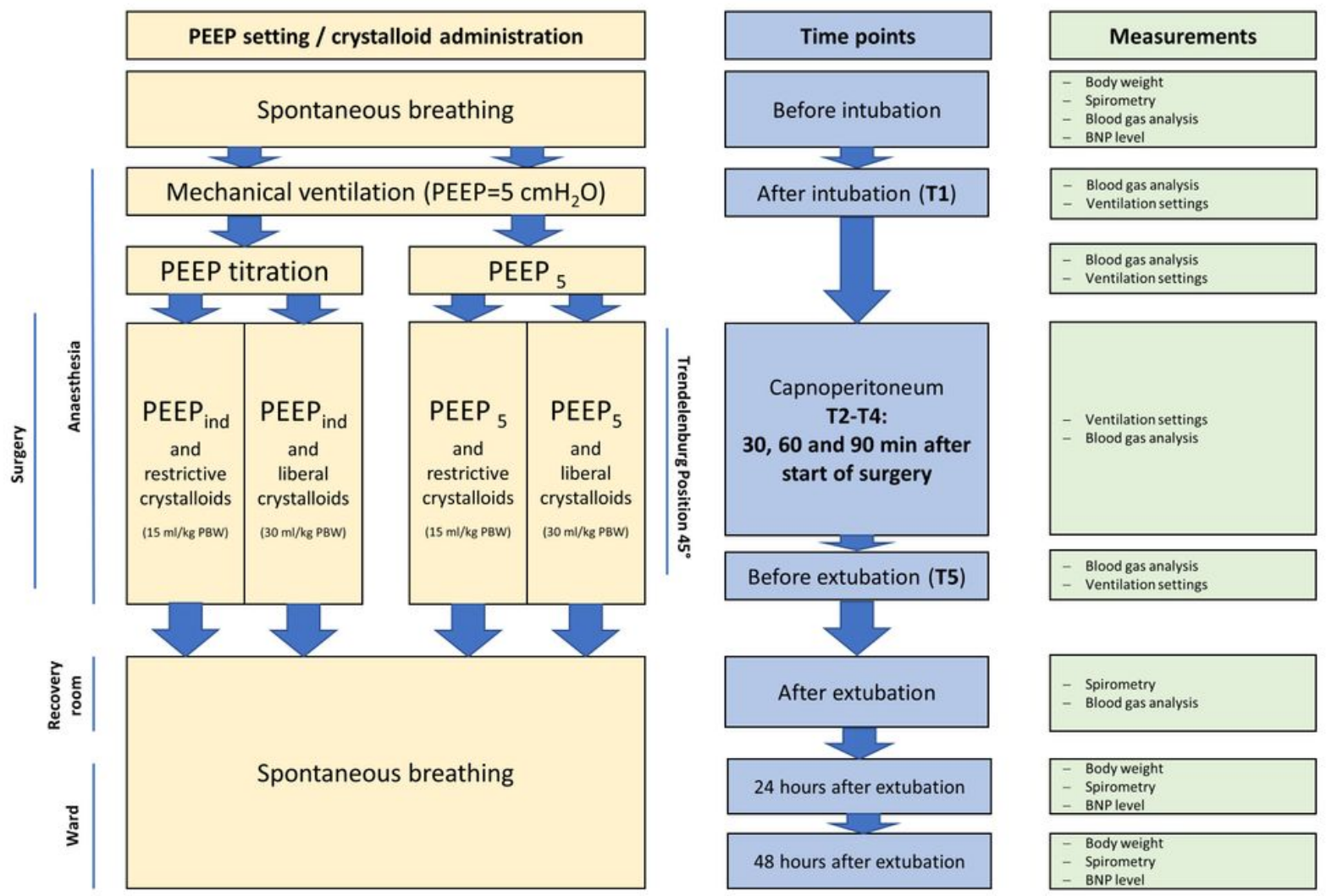

- Blood gas analysis

- Ventilation settings

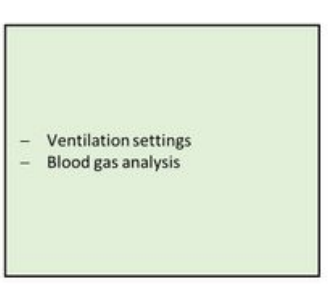

Blood gas analysis

Ventilation settings

After extubation

Spirometry

Blood gas analysis

\begin{tabular}{|l|} 
\\
\hline - Body weight \\
- Spirometry \\
- BNPlevel \\
\hline \\
\hline - Body weight \\
- Spirometry \\
- BNPlevel
\end{tabular}


Figure 2

Schematic diagram of the protocol and interventions for the study groups
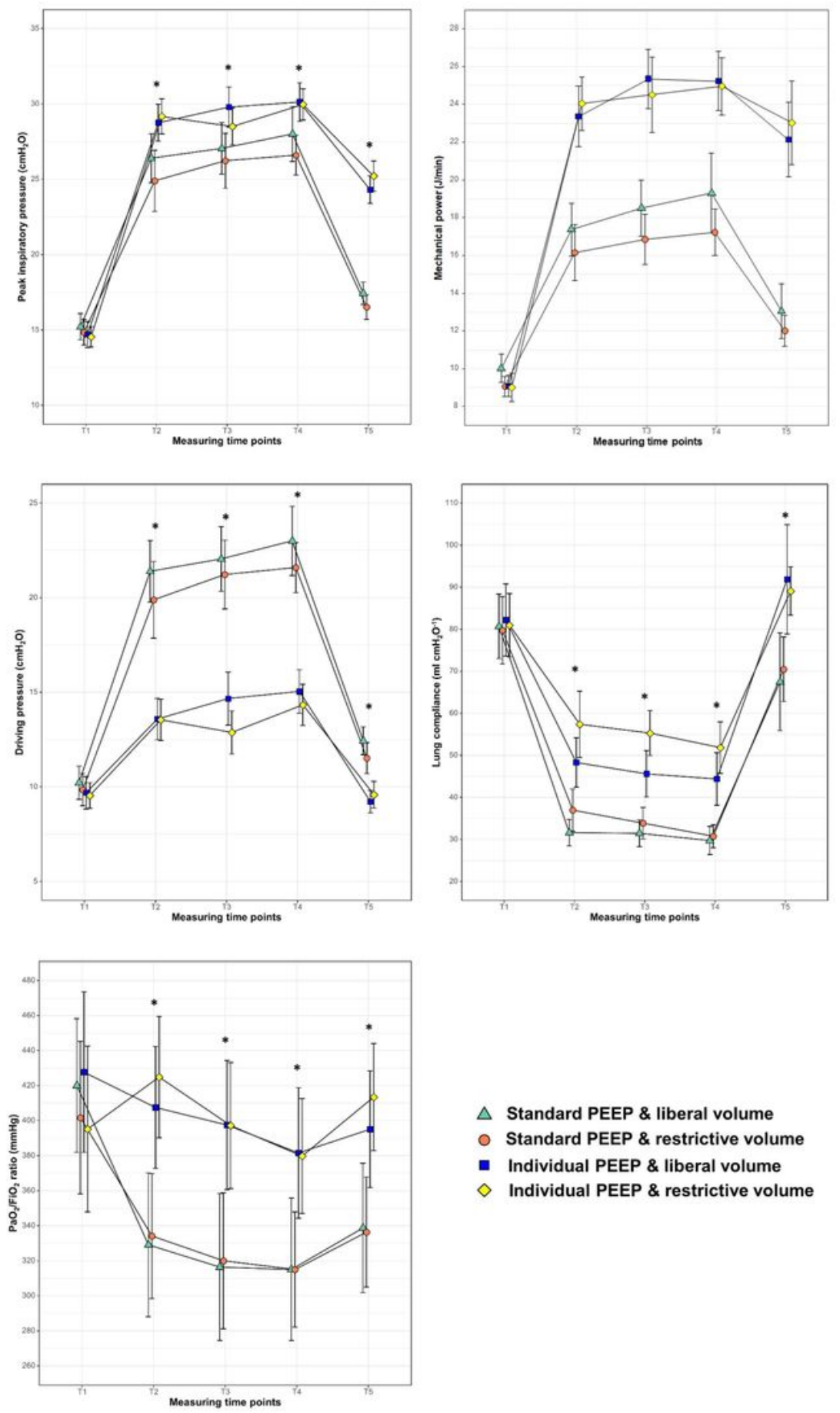
$\triangle$ Standard PEEP \& liberal volume
- Standard PEEP \& restrictive volume
- Individual PEEP \& liberal volume
$\diamond$ Individual PEEP \& restrictive volume

\section{Figure 3}

Differences in mechanical ventilation parameters between the study groups during robotic-assisted laparoscopic prostatectomy $(\mathrm{n}=98)$ Notes: ${ }^{*} \mathrm{P}<0.05$; Entries are the mean (SD), and P-values compare the 
four treatment arms at each timepoint using an ANOVA; FiO2: fraction of inspired oxygen; PaO2: partial pressure of oxygen in arterial blood; PBW: predicted body weight; individual PEEP: determined with titration; standard PEEP of $5 \mathrm{~cm} \mathrm{H} 2 \mathrm{O}$ was used; restrictive volume: $15 \mathrm{ml} / \mathrm{kg}$ PBW crystalloid solution until the end of surgery; liberal volume: $30 \mathrm{ml} / \mathrm{kg}$ PBW crystalloid solution until the end of surgery; T1: 5 min after intubation in supine position, T2: 30 min after start of capnoperitoneum and Trendelenburg position, T3: $60 \mathrm{~min}$ after start of capnoperitoneum and Trendelenburg position, T4: 90 min after start of capnoperitoneum and Trendelenburg position, T5: before extubation in supine position; mechanical power: calculated with the following formula: mechanical power $=0.098^{\star}$ tidal volume*respiratory rate* (PIP-0,5*driving pressure)

\section{Supplementary Files}

This is a list of supplementary files associated with this preprint. Click to download.

- TableS1.docx

- Tables2.docx 